\title{
Questes
}

Revue pluridisciplinaire d'études médiévales

Journée d'étude 1 - Trier, classer, organiser | 2022

Trier, classer, organiser

\section{Écriture et savoirs taxonomiques : quelques remarques}

Pierre Chastang

\section{(2) OpenEdition}

1 Journals

\section{Édition électronique}

URL : https://journals.openedition.org/questes/6078

DOI : 10.4000/questes.6078

ISSN : 2109-9472

Éditeur

Les Amis de Questes

Édition imprimée

Pagination : 258-265

ISSN : 2102-7188

\section{Référence électronique}

Pierre Chastang, «Écriture et savoirs taxonomiques : quelques remarques », Questes [En ligne], Journée d'étude 1 - Trier, classer, organiser | 2022, mis en ligne le 31 janvier 2022, consulté le 21 février 2022. URL : http://journals.openedition.org/questes/6078; DOI : https://doi.org/10.4000/ questes.6078

\section{(C) Association des amis de «Questes »}




\title{
Écriture et savoirs taxonomiques : quelques remarques
}

\author{
Pierre Chastang \\ DYPAC, UVSQ, Université Paris-Saclay
}

Si les historiens du Moyen Âge savent depuis très longtemps que les institutions pensent, comme le rappelait l'anthropologue Mary Douglas dans un essai paru en 1986, ils ont jusqu'alors peu questionné la manière dont cette pensée se formait, se diffusait, et se transmettait au travers d'artéfacts et de pratiques spécifiques. Il s'agit pourtant d'une question ancienne, qui façonne le questionnement sociologique de la fin du $x^{x}{ }^{e}$ siècle, en France comme en Allemagne quoique de manière différente $^{1}$. La pensée des individus, dans le monde social, dépend de classifications «toujours fournies déjà toutes faites en même temps que notre monde social ». Elles « stabilisent les flux de la vie sociale et créent même jusqu'à un certain point, les réalités auxquelles elles s'appliquent », en usant d'un pouvoir de qualification attaché à l'exercice de nomination où se dévoilent des systèmes de connaissance partagés, propre à un espace social. Les articles rassemblés proposent de ce point de vue un parcours, qui va du monde savant à celui des scriptoria ecclésiastiques, des commandes artistiques de l'Ecclesia aux milieux littéraires urbains.

Le présent volume, intitulé Trier, classer, organiser : ordonner le monde au Moyen Âge, rassemble les contributions de jeunes chercheurs

\footnotetext{
${ }^{1}$ Voir Mary Douglas, Comment pensent les institutions, suivi de La connaissance de soi et Il n'y a pas de don gratuit, Paris, La Découverte, 2004, p. 136-142.
} 
médiévistes au sujet de ces quatre opérations en se fondant sur l'analyse des artéfacts produits au Moyen Âge, qu'il s'agisse de textes ou d'images. Car la jonction disciplinaire opérée qui fait se côtoyer des contributions d'historiens, littéraires, et historiens de l'art révèle combien la question taxonomique au sens large est transverse aux découpages académiques et constitue un point de convergence et de confrontation des traditions méthodologiques. Question taxonomique au sens large car les textes ne s'intéressent pas uniquement aux processus de classifications stricto sensu, mais également aux procédures matérielles et intellectuelles qui les accompagnent, parmi lesquelles figurent le tri et l'ordonnancement d'objets, sans que l'on sache toujours s'il s'agit d'étapes distinctes ou de manières différentes de considérer un processus commun. Il en est ainsi par exemple des procédures de tri à l'œuvre dans la cartularisation des actes dans le cadre d'institutions ecclésiastiques locales qui apparaissent comme intimement liées à des enjeux de classification d'une matière archivistique à travers laquelle l'institution construit une temporalité et un espace social qui lui sont propres. Les contributions permettent également de prendre conscience que des dispositifs identiques du point de vue syntaxique, sémantique et documentaire, à l'image des listes de rois du haut Moyen Âge, peuvent avoir été produits pour des usages distincts.

Ainsi sont tour à tour convoquées des œuvres - textes et images qui relèvent des domaines de l'écrit pragmatique - diplomatique ou administratif -, savant, littéraire, mais également de la production d'images - figura et ornementum. À cette variété des types de documents correspond une pluralité dans la manière spécifique d'aborder la question taxonomique. Certains articles analysent des discours qui visent de manière explicite à produire des taxonomies s'appliquant au monde social ou aux domaines de la connaissance. Ils s'incarnent dans des formes 
discursives très variées - revues d'état, bestiaires ou encyclopédies - qui s'accompagnent de formes de publicisation et d'efficience elles-mêmes très diversifiées. Ces contributions s'inscrivent dans le renouveau du questionnement porté sur la stratification sociale médiévale ${ }^{2}$ qui, délaissant les catégorisations traditionnelles issues de l'histoire économique et sociale, introduit fortement la question du genre en écho avec certaines perspectives tracées par l'intersectionnalité anglosaxonne ${ }^{3}$. D'autres textes s'efforcent de confronter les catégories en usage dans la classification des végétaux et des animaux produite au Moyen Âge, en Orient et en Occident, dans un moment historique identifié par Philippe Descola comme relevant d'une ontologie analogiste ${ }^{4}$. Si des travaux récents ont interrogé la place des systèmes de classification des produits et des métiers dans le fonctionnement de l'économie de marché ${ }^{5}$, le présent volume propose une ouverture du spectre chronologique et déplace le questionnement vers ce qui pourrait constituer une épistémè prémoderne et vers l'économie des savoirs qu'elle induit.

Dans ce panorama des outils taxonomiques, la liste et secondairement le graphique arborescent tiennent une place centrale. Ils constituent des dispositifs sémantiques et graphiques utilisés par les scripteurs, et leur promotion en objets d'étude témoigne d'une prise en compte croissante, dans le travail des disciplines historiques, des recherches consacrées à l'histoire de la culture de l'écrit et des systèmes de communication anciens. Le changement du regard porté sur les

\footnotetext{
${ }^{2}$ Voir par exemple le dossier «Statuts sociaux»publié dans Annales. Histoire, Sciences sociales, vol. 68, $\mathrm{n}^{\mathrm{0}}$ 4, 2013.

${ }^{3}$ Voir le dossier «Les régimes de genre dans les sociétés occidentales de l'Antiquité au XVII siècle », Annales. Histoire, Sciences Sociales, vol. 67, n 3, 2012.

${ }^{4}$ Voir Philippe Descola, Par-delà nature et culture, Paris, Gallimard, 2005.

${ }^{5}$ Voir Nomenclatures et classifications : approches historiques, enjeux économiques, dir. Jérôme Bourdieu, Martin Bruegel et Alexandro Stanziani Versailles, INRA éd., 2004.
} 
sources de l'Histoire a en effet conduit à réévaluer, au détriment d'un paradigme du reflet et de l'enregistrement, la dimension performative de l'écrit. Elle a permis de percevoir l'action dans et sur le monde qu'induisent les opérations de nomination et d'inscription ${ }^{6}$, et de saisir le processus de scripturalisation du monde qui accompagne l'histoire de l'Occident à partir des siècles médians du Moyen Âge, ce dernier transformant de manière profonde non seulement les savoirs dans leur contenu, leur forme matérielle et leur dispositif cognitif, mais également un pouvoir qui s'incarne graduellement en un «réseau productif» (Michel Foucault) traversant le corps social ${ }^{7}$.

L'étude de formes graphiques et scripturales présentes dans la culture manuscrite du Moyen Âge ${ }^{8}$ conduit à faire l'expérience de l'indistinction médiévale entre mémoire «verbale» et mémoire « visuelle » à propos de laquelle Mary Carruthers écrit dans Machina memorialis : «Les lettres utilisées pour l'écriture avaient le même statut "visuel" que nos "images"; d'où il ressort que la page, l'ensemble du parchemin avec ses lettrines et ses ornements, était considérée comme une "image" à valeur cognitive ${ }^{9} »$. Cette visualité du texte apparaît toutefois comme étroitement liée à des formes rhétoriques que nous placerions du côté des savoirs discursifs, à commencer par ce que Mary

${ }^{6}$ Écriture et action: XVII ${ }^{e}$ XIX ${ }^{e}$ siècle, une enquête collective, dir. Groupe de Recherches Interdisciplinaires sur l'Histoire du Littéraire, Paris, Éditions de l'EHESS, 2016.

${ }^{7}$ Michel Foucault, «Intervista a Michel Foucault », dans Microfisica del potere. Interventi politici, dir. Alessandro Fontana et Pasquale Pasquino, Torino, G. Einaudi, 1977, p. 3-38 (repris en traduction française dans Michel Foucault, Dits et écrits, t. II, Paris, Gallimard, 2001, p. 140-160, ici p. 148-149).

${ }^{8}$ Pour une perspective globale sur cette question, voir Johanna Drucker, Graphesis. Visual Forms of Knowledge Production, Cambridge (Massachusetts)/London, Harvard University Press, 2014.

${ }^{9}$ Mary Carruthers, Machina memorialis. Méditation, rhétorique et fabrication des images au Moyen Âge, trad. Fabienne Durand-Bogaert, Paris, Gallimard, 2002 ; sur cette question voir également Vincent Debais, La Croisée des signes. L'écriture et les images médiévales (800-1200), Paris, Cerf, 2017. 
Carruthers propose de dénommer literacy pictures qui, sans être visibles, informent le texte et s'adressent directement à l'œil mental. La figure de l'arbor qui participe des deux formes de rhétorique - visuelle et discursive $\mathrm{e}^{10}$-, constitue un objet-frontière particulièrement captivant.

Dans l'inscription, ornements et graphiques, vides et pleins, couleurs et textures, narration et parataxe confèrent un rythme et un ordonnancement au texte et à la pensée; ils sont les vestiges d'une intelligence passée des formes de pensée comprises dans leur double dimension mentale et matérielle ${ }^{11}$. Ils constituent également les éléments à partir desquels s'édifient les systèmes taxonomiques partagés d'un texte à l'autre, d'un champ de l'espace social à l'autre. Dès lors, la matérialité des textes et, au-delà, des artéfacts culturels, se voit investie d'un enjeu qui dépasse largement le domaine dans lequel ils étaient jadis cantonnés. En scrutant avec attention formes, couleurs, signes et cadres, en décrivant avec précision l'agencement de l'écran de projection de la pensée ${ }^{12}$, les articles contribuent de manière convergente à réintroduire la question de la matérialité des objets et de la pensée, à rebours de la tradition ancienne et bien ancrée d'idéalité du texte. Ils ouvrent également à une compréhension plus fine des correspondances entre les formes intériorisées de la connaissance -dans lesquelles les techniques mnémoniques tiennent une place centrale - et celles matérialisées dans les livres par des textes, des images, ainsi que toutes les formes intermédiaires d'iconicité du texte et de littérarité de l'image.

${ }^{10}$ Sur cette question appliquée aux pratiques exégétiques, voir Patrice Sicard, Diagrammes médiévaux et exégèse visuelle. Le Libellus de formatione arche de Hugues de Saint-Victor, Turnhout, Brepols, 1993.

${ }^{11}$ Voir en dernier lieu, Jean-Claude Schmitt, Les Rythmes au Moyen Âge, Paris, Gallimard, 2016.

${ }^{12}$ Voir à ce sujet la notion de pensée de l'écran forgée par Anne-Marie Christin, L'Image écrite ou la déraison graphique, Paris, Flammarion, 2001. 
Dès lors, les médiévistes se retrouvent face à des questions importantes inscrites dans la tradition sociologique depuis l'époque de ses pionniers : celle en particulier des rapports entre taxonomies autochtones et taxonomies savantes ${ }^{13}$, l'investigation des catégories en usage au Moyen Âge contribuant à renforcer les perspectives d'une histoire critique qui s'interroge sur nos propres cadres d'interprétation. Les travaux de Jack Goody, La Raison graphique en particulier, constituent de ce point de vue un héritage vivant, dans la mesure où l'attention qu'il a portée sur les étapes historiques de l'avènement de l'écriture comme « technologie de l'intellect » et son influence sur les systèmes culturels et sociaux, s'est accompagnée du développement d'une réflexion critique portée sur les opérations et outils heuristiques de l'anthropologue, à commencer par le tableau et la liste ${ }^{14}$.

Cette dernière forme d'écriture est très présente dans les contributions rassemblées dans ce volume, sans doute parce qu'elle constitue un dispositif omniprésent dans la culture de l'écrit du Moyen Âge, aussi bien dans les textes pragmatiques, poétiques que didactiques ${ }^{15}$. Mais au-delà de cette forte densité des «textes à listes », elle constitue une voie d'étude féconde des savoirs taxonomiques, dans la mesure où la liste apparaît comme une forme commune aux domaines de l'administration, des sciences de la nature et des sciences du texte, considérés dans le temps long. Elle constitue également une forme

13 Émile Durkheim et Marcel Mauss, «De quelques formes primitives de classification. Contribution à l'étude des représentations collectives », Année sociologique, 6, 1903, p. 1-72.

${ }^{14}$ Sur l'introduction d'une conscience littéraire dans la pratique anthropologique, voir Writing Culture: The Poetics and Politics of Ethnography, dir. James Clifford et George E. Marcus, Berkeley, University of California Press, 1986.

${ }^{15}$ Voir par exemple Liste et effet liste en littérature, dir. Michelle Lecolle et Sophie Milcent-Lawson, Paris, Classiques Garnier, 2013 et le programme ANR POuvoir des LIstes au Moyen Âge, dirigé par Pierre Chastang et Laurent Feller [http://polima.huma-num.fr]. 
d'écriture syntaxique, graphique et sémantique qui crée un dispositif complexe situé à la frontière du verbal et du visuel. On constate, dans la variété des études de cas rassemblées dans le présent volume, le caractère ductile de la liste qui s'adapte à des situations et des visées très disparates, et se présente d'ailleurs sous des formes scripturales ellesmêmes hétérogènes. Elle constitue en revanche un véritable outil de saisie et d'ordonnancement des choses, au-delà même de la mise en liste comme dispositif scriptural. La pensée par listes pourrait être définie comme un habitus intellectuel, tributaire de formes d'« ornementation mentale » (Mary Carruthers). La multiplication des contextes sociaux d'usage permet, à rebours de la recherche d'une efficience cognitive globale, d'évaluer la souplesse de l'outil et de révéler des formes d'intertextualité qui dessinent les contours de circulations, de rapports d'autorité, mais également de jeux linguistiques et verbaux dans lesquels le décalage perçu par les lecteurs/auditeurs fait sens. C'est aussi à cerner les pouvoirs particuliers de ces outils sémantico-graphiques que les contributions s'emploient. Elles pointent tour à tour les pouvoirs de démarcation et de canonisation ${ }^{16}$ attachés à son usage, sa double valeur de cardinalité et d'ordinalité, qui permet de hiérarchiser et de compter, son pouvoir enfin de décontextualisation, mis en évidence par Jack Goody, qui ouvre la voie à des pratiques d'extraction et de recombinaison des éléments assemblés. En creux, apparaissent la présence et la circulation des savoirs comptables au Moyen Âge à propos desquels Jacqueline Cerquiglini avait attiré l'attention il y a plusieurs années déjà : «le "dit" conte/compte et devise/divise ». Si en latin classique computare a le sens de compter, il signifie également en latin médiéval rendre des comptes

\footnotetext{
${ }^{16}$ Sur cette dernière notion et sa valeur culturelle, voir Jan Assmann, La Mémoire culturelle. Écriture, souvenir et imaginaire politique dans les civilisations antiques, Paris, Aubier, 2010, p. 93, 94-95, 96, 100 et 111.
} 
(rationem reddere) et conter (narrare ${ }^{17}$ ). C'est bien à une poétique des savoirs taxonomiques que cette conjonction de significations nous invite. Elle ne peut être menée que dans le cadre interdisciplinaire esquissé tout au long de cet ouvrage collectif.

17 Jacqueline Cerquiglini-Toulet, «Le Dit», dans Grundriß der romanischen Literaturen, t. 8/1 : «La littérature française aux $\mathrm{XIV}^{\mathrm{e}}$ et $\mathrm{XV}^{\mathrm{e}}$ siècles », dir. Daniel Poirion, Heidelberg, C. Winter, 1988, p. 86-94. 